\title{
A LOEWNER-TYPE LEMMA FOR WEIGHTED BIHARMONIC OPERATORS
}

\author{
Miroslav EnGLIŠ
}

In the present note we give a simpler proof of the recent result of Hedenmalm that the Green function for the weighted biharmonic operator $\Delta|z|^{2 \alpha} \Delta, \alpha>-1$, on the unit disc $\mathbf{D}$ with the Dirichlet boundary conditions is positive. The main ingredient, which in the special case of the unweighted biharmonic operator $\Delta^{2}$ is due to Loewner and which is of an independent interest, is a lemma characterizing, for a positive $C^{2}$ weight function $w$, the second-order linear differential operators which take any function $u$ satisfying $\Delta w^{-1} \Delta u=0$ into a harmonic function. Another application of this lemma concerning positivity of the Poisson kernels for the biharmonic operator $\Delta^{2}$ is also given.

Let $\Omega$ be a bounded domain in the complex plane with smooth boundary, $w$ a function which is $C^{2}$-continuous and positive on $\bar{\Omega}$ except for a finite set $S$ of isolated singularities in $\Omega$, where it can have a zero or become $+\infty$ (or even not be defined at all); such functions will be termed weights. We will be interested in the weighted biharmonic operators $\Delta w^{-1} \Delta$ on $\Omega$, where $\Delta$ stands for the Laplacian. For brevity, let us call the above $w$ biharmonic operators, and similarly a function $u$ satisfying $\Delta w^{-1} \Delta u=0$ will be called a $w$-biharmonic function. The latter means, by definition, that $u$ is a $C^{2}$ function on $\Omega \backslash S$ such that $w^{-1} \Delta u$ extends by continuity to a harmonic function on all of $\Omega$. The $w$-biharmonic Green function $U(x, y)$ is the solution to the boundary value problem

$$
\begin{cases}\Delta_{x} \frac{1}{w(x)} \Delta_{x} U(x, y)=\delta(x-y) & \text { (the delta function), } \\ U(x, y)=\frac{\partial U}{\partial n_{x}}(x, y)=0 & \text { for } x \in \partial \Omega,\end{cases}
$$

where $\partial / \partial n$ denotes differentiation in the outward normal direction. The first equation must again be understood in the sense that, for fixed $y, U$ is in $C^{2}(\Omega \backslash S \backslash\{y\})$ and $w^{-1} \Delta U=\Gamma(\cdot, y)+h_{y}$ where $\Gamma(x, y)$ is the ordinary Green function (for the Laplace operator) on $\Omega$ and $h_{y}$ is harmonic on all of $\Omega$. Quite recently, a lot of attention has been attracted by the problem of positivity of these Green functions, for various weights $w$. The motivation 
for this comes mainly from applications in the theory, initiated by Hedenmalm, of contractive zero divisors and factorization of functions in Bergman spaces, cf. [DKSS1], [DKSS2], [H-jram], [H-OpP]. For $w \equiv 1$, i.e. the unweighted biharmonic operator, the Green function is related to bending of a clamped plate and to the creeping flow on $\Omega$, and the question concerning its positivity comes back to Hadamard and Boggio (see the discussion in [H-Duke] for more details and references). In that case, the answer is known to be positive for a few domains (e.g. a disc, or Pascal's limaçon) and negative for many others (sufficiently eccentric ellipses [Gar], [ShT], sufficiently elongated rectangles [NS], all annuli [E-Pee]; also, granted we relax the condition that $\Omega$ be bounded, the infinite strip [Duff]). For general weight $w$ and $\Omega$ the unit disc $\mathbf{D}$, various results were obtained by Hedenmalm [H-Duke], [H-OpP], Shimorin [Shi1], [Shi2], and Stessin [Ste]; see also [H-BVP]. Observe that, if $\Omega$ is simply connected and $\phi$ is a conformal map of $\mathbf{D}$ onto $\Omega$, the pullback via $\phi$ of the Green function for $\Delta^{2}$ on $\Omega$ is the Green function for $\Delta\left|\phi^{\prime}\right|^{-2} \Delta$ on $\mathbf{D}$; thus, some of the results just mentioned (i.e., for general weight $w$ on $\mathbf{D}$ ) can also be interpreted as results about unweighted biharmonic operators on different domains (which are images of D under a conformal map).

The main result of [H-Duke] is a proof of the positivity of the Green function $U$ for the operator $\Delta|z|^{-2 \alpha} \Delta, \alpha>-1$, on $\mathbf{D}$. Although an explicit formula for $U$ is given for $\alpha$ an integer ([H-Duke], Theorem 4.6), the positivity is not completely obvious directly from it; instead, it is obtained for all $\alpha$ as a consequence of the positivity of the Green function for the operator $\Delta \exp (-2 \alpha \operatorname{Im} z) \Delta$ on the upper half-plane, which in turn is reduced, by a skillful and quite involved explicit computation using the above-mentioned formula for integer $\alpha$, to the positivity of a certain integral, and the last finally follows by an argument based on Pólya's lemma (see [H-Duke, p. 71]). The purpose of this note is to present a simpler proof, which, essentially, uses little beyond the ordinary maximum principle for harmonic functions. Its basic ingredient is the following lemma, which may be of some independent interest and whose special case $w \equiv 1$ goes essentially back to Charles Loewner [Loe].

Lemma 1 (Fundamental Lemma). Assume that $w$ is $C^{2}$ and positive on an open set $\mathcal{V}$. Let $m$ be a function on $\mathcal{V}$ satisfying

$$
\begin{gathered}
\frac{1}{w} \Delta m=\text { const. } \\
\partial \frac{1}{w} \partial m=\bar{\partial} \frac{1}{w} \bar{\partial} m=0
\end{gathered}
$$


and form the second-order linear differential operator

$$
\mathcal{L} u:=\frac{1}{w}[m \Delta u-\nabla m \cdot \nabla u+u \Delta m] .
$$

Then $\mathcal{L}$ transforms every $w$-biharmonic function on $\mathcal{V}$ into a harmonic function. Conversely, every second order linear differential operator sending an arbitrary $w$-biharmonic function on $\mathcal{V}$ into a harmonic one must be of the form (2) with $m$ satisfying (1).

Here and elsewhere, $\nabla m \cdot \nabla u$ stands for the scalar product of the complex gradients

$$
\nabla m \cdot \nabla u=\partial m \cdot \bar{\partial} u+\bar{\partial} m \cdot \partial u
$$

where $\partial \equiv \partial / \partial z, \bar{\partial} \equiv \partial / \partial \bar{z}$ (the Wirtinger operators). Also we employ a slightly nonstandard definition of the Laplacian

$$
\Delta:=\partial \bar{\partial}=\frac{1}{4}\left(\frac{\partial^{2}}{\partial x^{2}}+\frac{\partial^{2}}{\partial y^{2}}\right) \quad(z=x+y i)
$$

which differs from the usual one by a factor of 4 .

Proof. We begin with the converse part. Assume that $\mathcal{L}$, which we choose to write in the form

$$
\mathcal{L} u=\frac{1}{w}[m \partial \bar{\partial} u+b \partial \partial u+c \bar{\partial} \partial u]+A \partial u+B \bar{\partial} u+C u,
$$

sends an arbitrary $w$-biharmonic function into a harmonic one. Expanding we have

$$
\Delta \mathcal{L} u=m \Delta \frac{1}{w} \Delta u+b \Delta \frac{1}{w} \partial \partial u+c \Delta \frac{1}{w} \overline{\partial \partial} u+\text { terms of lower order. }
$$

Since the only relation between the derivatives of $u$ up to the fourth order is given by $\Delta w^{-1} \Delta u=0$, we see already from (4) that

$$
b=c=0,
$$

and we can thus write (3) in the form

$$
\mathcal{L} u=\frac{1}{w} m \Delta u+A \partial u+B \bar{\partial} u+C u .
$$

Hence we have

$$
\begin{aligned}
\Delta \mathcal{L} u=\Delta & m \cdot \frac{1}{w} \Delta u+m \cdot \Delta \frac{1}{w} \Delta u+\partial m \cdot \bar{\partial} \frac{1}{w} \Delta u+\bar{\partial} m \cdot \partial \frac{1}{w} \Delta u \\
& +\Delta A \cdot \partial u+A \cdot \Delta \partial u+\partial A \cdot \Delta u+\bar{\partial} A \cdot \partial \partial u \\
& +\Delta B \cdot \bar{\partial} u+B \cdot \Delta \bar{\partial} u+\bar{\partial} B \cdot \Delta u+\partial B \cdot \bar{\partial} \partial \\
& +\Delta C \cdot u+C \cdot \Delta u+\partial C \cdot \bar{\partial} u+\bar{\partial} C \cdot \partial u
\end{aligned}
$$


Here we have used the formula

$$
\Delta(f g)=f \Delta g+g \Delta f+\partial f \cdot \bar{\partial} g+\bar{\partial} f \cdot \partial g
$$

which will be of great service to us also later on.

Now, first of all, $\Delta \mathcal{L} u$ must vanish for harmonic $u$. For $\Delta u=0$, the right-hand side of (6) reduces to

$$
\Delta A \cdot \partial u+\bar{\partial} A \cdot \partial \partial u+\Delta B \cdot \bar{\partial} u+\partial B \cdot \bar{\partial} \partial+\Delta C \cdot u+\partial C \cdot \bar{\partial} u+\bar{\partial} C \cdot \partial u .
$$

Since the only relation between the derivatives of $u$ up to the second order is $\Delta u=0$, we must have

$$
\Delta A+\bar{\partial} C=0, \quad \Delta B+\partial C=0, \quad \Delta C=0, \quad \bar{\partial} A=0, \quad \partial B=0 .
$$

From the last two conditions we see that $\Delta A=\Delta B=0 ;$ the first two then give $\partial C=\bar{\partial} C=0$, and we conclude that

$$
\begin{aligned}
C & \equiv \text { const. } \\
\bar{\partial} A & =\partial B=0 .
\end{aligned}
$$

Feeding this back into (6) and using again the formula (7), we obtain

$$
\begin{aligned}
& \Delta \mathcal{L} u=\Delta m \cdot \frac{1}{w} \Delta u+m \cdot \overbrace{\Delta \frac{1}{w} \Delta u}^{=0}+ \\
& +\partial m \cdot \bar{\partial}\left(\frac{1}{w}\right) \cdot \Delta u+\partial m \cdot \frac{1}{w} \bar{\partial} \Delta u+\bar{\partial} m \cdot \partial\left(\frac{1}{w}\right) \cdot \Delta u+\bar{\partial} m \cdot \frac{1}{w} \partial \Delta u+ \\
& +A \cdot \Delta \partial u+B \cdot \Delta \bar{\partial} u+(\partial A+\bar{\partial} B) \cdot \Delta u+C \cdot \Delta u \text {. }
\end{aligned}
$$

Comparing the coefficients at $\Delta \partial u, \Delta \bar{\partial} u$, and $\Delta u$, respectively, we see that for $\Delta \mathcal{L} u$ to vanish for arbitrary $w$-biharmonic function $u$ it is necessary that

$$
\begin{gathered}
A+\frac{1}{w} \bar{\partial} m=0, \quad B+\frac{1}{w} \partial m=0 \\
\frac{1}{w} \Delta m+\partial m \cdot \bar{\partial}\left(\frac{1}{w}\right)+\bar{\partial} m \cdot \partial\left(\frac{1}{w}\right)+\partial A+\bar{\partial} B+C=0 .
\end{gathered}
$$

From (12) we have

$$
\begin{aligned}
\partial A & =-\partial\left(\frac{1}{w}\right) \cdot \bar{\partial} m-\frac{1}{w} \Delta m, & \bar{\partial} B & =-\bar{\partial}\left(\frac{1}{w}\right) \cdot \partial m-\frac{1}{w} \Delta m, \\
\bar{\partial} A & =-\bar{\partial} \frac{1}{w} \bar{\partial} m, & \partial B & =-\partial \frac{1}{w} \partial m,
\end{aligned}
$$


so we may rewrite (13) as

$$
0=\frac{1}{w} \Delta m-\frac{1}{w} \Delta m-\frac{1}{w} \Delta m+C
$$

or

$$
\frac{1}{w} \Delta m=C
$$

and (10) as

$$
0=\bar{\partial} \frac{1}{w} \bar{\partial} m=\partial \frac{1}{w} \partial m .
$$

Our operator $m$ must therefore be of the form

$$
\mathcal{L} u=\frac{1}{w} m \Delta u-\frac{1}{w} \bar{\partial} m \cdot \partial u-\frac{1}{w} \partial m \cdot \bar{\partial} u+\frac{1}{w} \Delta m \cdot u,
$$

which is (2), with $m$ satisfying (9), (14), and (15), which is (1).

Conversely, let $\mathcal{L}$ be any operator of the form (5), with $A$ and $B$ given by (12), which satisfies (9), (14), and (15). Then it follows from the formulas (6) and (11) that

$$
\Delta \mathcal{L} u=m \cdot \Delta \frac{1}{w} \Delta u
$$

and so $\mathcal{L}$ sends an arbitrary $w$-biharmonic function into a harmonic one. This completes the proof.

Corollary 2. If $m$ is a real-valued function satisfying

$$
\Delta m=w, \quad \partial \frac{1}{w} \partial m=0,
$$

then the operator (2) transforms w-biharmonic functions into harmonic ones.

Indeed, for real-valued $m, \bar{\partial} w^{-1} \bar{\partial} m=\overline{\partial w^{-1} \partial m}=0$, and so (1) is fulfilled. As an application, we prove the promised positivity result for a weighted biharmonic Green function.

Theorem 3. Let $\alpha>-1$. Then the Green function for the weighted biharmonic operator $\Delta|z|^{-2 \alpha} \Delta$ on the unit disc $\mathbf{D}$ is positive.

Proof. Since $w=|z|^{2 \alpha}$, the hypotheses (1) of the Fundamental Lemma (or, rather, of its Corollary) are satisfied for

$$
m=\frac{|z|^{2(\alpha+1)}-1}{(\alpha+1)^{2}}
$$


on the open set $\mathcal{V}=\mathbf{D} \backslash\{0\}$ where $w$ is $C^{2}$ and positive. Indeed, $\Delta m=$ $(\alpha+1)^{-2} \partial \bar{\partial}\left(z^{\alpha+1} \bar{z}^{\alpha+1}-1\right)=w$, and

$$
\partial \frac{1}{w} \partial m=\partial\left(\frac{1}{|z|^{2 \alpha}} \cdot \frac{\bar{z}|z|^{2 \alpha}}{\alpha+1}\right)=\frac{1}{\alpha+1} \partial \bar{z}=0 .
$$

Hence, on $\mathbf{D} \backslash\{0\}$, the conclusion of the Lemma holds true for the operator

$$
\begin{aligned}
\mathcal{L} u & =\frac{m}{w} \Delta u-\left(\frac{\partial m}{w} \bar{\partial} u+\frac{\bar{\partial} m}{w} \partial u\right)+u \\
& =\frac{m}{w} \Delta u-\frac{1}{\alpha+1}(\bar{z} \bar{\partial} u+z \partial u)+u .
\end{aligned}
$$

Suppose now to the contrary that the Green function $U(x, y) \leq 0$ for some $x, y \in \mathbf{D}$. Since the function $U(\cdot, y)$ vanishes on $\partial \mathbf{D}$, it must attain its minimum at some interior point $x_{0} \in \mathbf{D}$, and $U\left(x_{0}, y\right) \leq 0$. Let us take $U(\cdot, y)$ as the function $u$ in (16). The function $v:=\mathcal{L} u$ is then harmonic on $\mathbf{D} \backslash\{0, y\}$. We claim that it is, in fact, harmonic on all of $\mathbf{D} \backslash\{y\}$. Indeed, in view of the definition of $u$,

$$
\frac{1}{w(z)} \Delta u(z)=\log \left|\frac{z-y}{1-\bar{y} z}\right|^{2}+h_{y}(z)
$$

with $h_{y}$ harmonic on $\mathbf{D}$. Thus, if $y \neq 0$,

$$
\Delta u(z)=|z|^{2 \alpha} \cdot \text { a function real-analytic at the origin. }
$$

Consequently,

$$
u(z)=|z|^{2(\alpha+1)} f_{1}(z)+h(z)
$$

and

$$
\partial u(z)=\bar{z}|z|^{2 \alpha} f_{2}(z)+f_{3}(z)
$$

with $f_{1}(z), f_{2}(z), f_{3}(z)$ real-analytic at the origin and $h(z)$ harmonic on $\mathbf{D}$. In particular, as $\alpha>-1$, it follows that $u(z), z \partial u(z)$ and $\bar{z} \bar{\partial} u(z)=\overline{z \partial u(z)}$ are continuous at the origin, and, consequently, so is $v$. By the removable singularities theorem for harmonic functions, the harmonicity of $v$ on $\mathbf{D} \backslash$ $\{0, y\}$ therefore implies that $v$ is harmonic on all of $\mathbf{D} \backslash\{y\}$.

Now on the unit circle, both $u$ and $\nabla u$ vanish (by the definition of the Green function) and so does $m$; hence, $v=0$ on $\partial \mathbf{D}$. At $y, u$ and $\bar{z} \bar{\partial} u+z \partial u$ are continuous, while $w(z)^{-1} \Delta u(z) \sim \log |z-y|^{2}$ tends to $-\infty$; it follows 
that $v(y)=+\infty$. By the maximum principle, $v \geq 0$ in $\mathbf{D} \backslash\{y\}$, and actually $v>0$ since an equality at any point would imply that $v \equiv 0$, contradicting the fact that $v$ have a logarithmic singularity at $y .{ }^{1}$ Thus, in particular, $v\left(x_{0}\right)>0$; since $u\left(x_{0}\right) \leq 0$ by assumption and, $x_{0}$ being a local minimum of $u, \nabla u\left(x_{0}\right)$ vanishes, we get

$$
0<v\left(x_{0}\right)=u\left(x_{0}\right)+\frac{m}{w} \Delta u\left(x_{0}\right) \leq \frac{m}{w} \Delta u\left(x_{0}\right) .
$$

If $\underline{x_{0} \neq 0}$, this implies $\Delta u\left(x_{0}\right)<0\left(\Delta u\left(x_{0}\right)=-\infty\right.$ if $\left.x_{0}=y\right)$, which is contradictory to $x_{0}$ being a local minimum of $u$. So $x_{0}=0$. That is, the only place where $U(\cdot, y), y \in \mathbf{D}$, can have a local minimum is the origin. Owing to the symmetry of the Green function $(U(z, y)=U(y, z))$, we can apply the same reasoning to the function $u(y)=U(y, 0)=U(0, y)$, which leads to the conclusion that if $U(x, y) \leq 0$ for some $x, y \in \mathbf{D}$, then $U$ must have its global minimum at the point $(0,0)$.

On the other hand, by the definition of the Green function we have

$$
\Delta_{z} U(z, 0)=|z|^{2 \alpha}\left(\log |z|^{2}+h_{0}(z)\right)
$$

for some harmonic function $h_{0}$. In view of the rotational symmetry, $h_{0}(z)$ depends only on the modulus $|z|$, and so must be a constant function. An easy integration therefore gives

$$
U(z, 0)=\frac{1}{(\alpha+1)^{2}}|z|^{2(\alpha+1)}\left(\log |z|^{2}-\frac{2}{\alpha+1}+h_{0}\right)+h_{1}(z)
$$

with another harmonic function $h_{1}$ (which again reduces to a constant, for the same reason). Thus

$$
U(r, 0)-U(0,0)=(\alpha+1)^{-2} r^{2(\alpha+1)}\left(\log r^{2}+h_{0}-\frac{2}{\alpha+1}\right)<0
$$

for sufficiently small $r>0$, which however shows that $U(0,0)$ cannot be a local - not to say global - minimum for $U(\cdot, 0)$. Thus we have arrived at a contradiction, which completes the proof.

If $\phi$ is a biholomorphic mapping of $\Omega_{1}$ onto $\Omega_{2}$ and $G$ is the Green function for $\Delta w^{-1} \Delta$ on $\Omega_{2}$, it is easily seen that $G \circ \phi$ must be the Green function for $\Delta\left[\left|\phi^{\prime}\right|^{2}(w \circ \phi)\right]^{-1} \Delta$ on $\Omega_{1}$. Using conformal mapping, we therefore immediately obtain the following result.

Corollary 4. Let $\Omega$ be a simply connected domain and $\phi$ a Riemann mapping of $\Omega$ onto $\mathbf{D}$. Then the Green function for the operator $\Delta\left[\left|\phi^{\prime}\right|^{2}\right.$. $\left.|\phi|^{2 \alpha}\right]^{-1} \Delta, \alpha>-1$, on $\Omega$ is positive.

\footnotetext{
${ }^{1}$ In fact, one must have, quite explicitly, $v(z)=m(y) \log |(z-y) /(1-\bar{y} z)|^{2}$.
} 
In particular, the Green function for the operator

$$
\Delta\left|(z-a)^{\alpha} /(1-\bar{a} z)^{\alpha+2}\right|^{-2} \Delta \quad(a \in \mathbf{D}, \alpha>-1)
$$

on $\mathbf{D}$ is positive.

We conclude by another result which can be obtained along the same vein. Recall that the function

$$
D(\xi, y):=\frac{1}{w(\xi)} \Delta_{\xi} U(\xi, y), \quad \xi \in \partial \Omega, y \in \Omega
$$

plays the role of the Poisson kernel for the operator $\Delta w^{-1} \Delta$. That is, for $f$ a function on $\partial \Omega$, the function

$$
F(y):=-\int_{\partial \Omega} f(\xi) D(\xi, y) d \sigma(\xi)
$$

is $w$-biharmonic in $\Omega$ and has boundary values $F=0, \partial F / \partial n=f$. In other words, $D(\xi, y)$ solves the boundary value problem

$$
\begin{cases}\Delta_{y} \frac{1}{w(y)} \Delta_{y} D(\xi, y)=0, & y \in \Omega, \\ D(\xi, y)=0, & y \in \partial \Omega, \\ \frac{\partial}{\partial n_{y}} D(\xi, y)=-\delta(y-\xi), & y \in \partial \Omega .\end{cases}
$$

(See e.g. [E-Pee], Section 5, or [H-Duke], [H-BVP].) It is easy to see that if $U(x, y) \geq 0$ on $\Omega \times \Omega$, then $D(\xi, y) \geq 0$ on $\partial \Omega \times \Omega$. In fact, $D(\xi, y)<0$ implies that $\frac{\partial^{2}}{\partial n_{\xi}^{2}} U(\xi, y)=\Delta_{\xi} U(\xi, y)=w(\xi) D(\xi, y)<0$, and as $U(\xi, y)=$ $\frac{\partial}{\partial n_{\xi}} U(\xi, y)=0$, it would follow that $U(x, y)<0$ for all $x$ lying sufficiently close to $\xi$ on the inward normal from $\xi$. It is an open question whether $D(\xi, y)>0$ on $\partial \Omega \times \Omega$ does not actually imply that already $U(x, y)>0$ on $\Omega \times \Omega$. The general opinion seems to be that more likely this is not the case; a small contribution in favour of the opposite is provided by the assertion below. Loosely speaking, its sense is that if $U(x, y)$ has a negative minimum for $x$ "not far" from $y$, then $D(\cdot, y)$ has to take negative values somewhere on the boundary.

Proposition 5. Let $\Omega$ be a bounded domain whose boundary consists of finitely many Jordan curves and let $U(x, y)$ be the Green function for the unweighted biharmonic operator $\Delta^{2}$ on $\Omega$. If $U(x, y)<0$ is a local minimum 
for some $x, y$ which lie in a disc $B\left(z_{0}, r\right)$ contained wholly in $\Omega$, then there is $\xi \in \partial \Omega$ for which $D(\xi, y)<0$.

Proof. Since $w \equiv 1$, we can take

$$
m(z)=\left|z-z_{0}\right|^{2}-r^{2}
$$

as our function $m$, and $u(z)=U(z, y)$ as the function $u$ in (2). This choice of $m$ satisfies

$$
m(y)<0, \quad m>0 \text { on } \partial \Omega, \quad m(x)<0 .
$$

From the Fundamental Lemma we conclude that

$$
v(z):=m(z) \Delta_{z} U(z, y)-\nabla m \cdot \nabla_{z} U(z, y)+U(z, y)
$$

is harmonic on $\Omega \backslash\{y\}$. For $z=x, v(x)=m(x) \Delta_{x} U(x, y)+U(x, y)$ is negative by assumption. Since $v(y)=+\infty$ (by a similar argument as in the proof of Theorem 3), we must have $v(\xi)<0$ at some point $\xi$ on $\partial \Omega$. But in view of the definition of the Green function, $v(z)=m(z) \Delta_{z} U(z, y)=m(z) D(z, y)$ on $\partial \Omega$, so $D(\xi, y)<0$ as well.

We close with a few comments regarding the solvability of the system (1):

$$
\begin{gathered}
\frac{1}{w} \Delta m=C, \\
\partial \frac{1}{w} \partial m=\bar{\partial} \frac{1}{w} \bar{\partial} m=0,
\end{gathered}
$$

where $C$ is a complex constant. Obviously, $m \equiv$ const. is a solution for any $w$. On the other hand, we have seen that for $w=\left|f^{\prime}\right|^{2}$, with $f$ a holomorphic function, one gets a nontrivial solution $m=|f|^{2}$. It turns out that these are essentially the only possibilities that can happen.

Proposition 6. Let $w$ be positive and $C^{2}$ on a simply connected open set $\mathcal{V}$. Then the system (17) has nontrivial solutions if and only if the function $\log w$ is harmonic. In that case, we can write $w=\left|H^{\prime}\right|^{2}$ where $H$ is a holomorphic function on $\mathcal{V}$ whose derivative does not vanish, and all solutions to (17) are given by

$$
m=C|H|^{2}+\alpha H+\beta \bar{H}+\gamma,
$$

where $\alpha, \beta, \gamma$ are arbitrary complex numbers.

Proof. Let $m$ be a solution of (17). Owing to the second equation in (17), we must have

$$
\partial m=\bar{f} w, \quad \bar{\partial} m=g w
$$


for some functions $f, g$ holomorphic on $\mathcal{V}$. Applying $\bar{\partial}$ to the first and $\partial$ to the second equation and using the first equation in (17), we obtain

$$
\bar{f} \frac{\bar{\partial} w}{w}+\overline{f^{\prime}}=g \frac{\partial w}{w}+g^{\prime}=C .
$$

If $f=g \equiv 0,(18)$ shows that $m$ is a constant; so let us assume that, for instance, $g$ is not identically zero. Then (19) gives

$$
\frac{\partial w}{w}=\frac{C-g^{\prime}}{g} .
$$

The right-hand side is a meromorphic function on $\mathcal{V}$, whereas the left-hand side is continuous by hypothesis. Hence $\partial w / w$ must be a holomorphic function on $\mathcal{V}$, and so

$$
0=\bar{\partial} \frac{\partial w}{w}=\bar{\partial}(\partial \log w)=\Delta \log w
$$

as asserted.

Conversely, if $\log w$ is harmonic, then owing to the simple connectivity of $\mathcal{V}$ we can write $\log w=F+\bar{F}$ with $F$ holomorphic on $\mathcal{V}$. Thus $w=\left|H^{\prime}\right|^{2}$ where $H$ is any primitive of the holomorphic function $e^{F}$. Since $\Delta|H|^{2}=w$, any solution $m$ to (17) must satisfy

$$
m=C|H|^{2}+f+\bar{g}
$$

for some holomorphic functions $f$ and $g$. Feeding this into the second equation in (17) gives

$$
\frac{1}{H^{\prime}} \overline{\left(\frac{g^{\prime}}{H^{\prime}}\right)^{\prime}}=\frac{1}{\overline{H^{\prime}}}\left(\frac{f^{\prime}}{H^{\prime}}\right)^{\prime}=0
$$

so both $f^{\prime} / H^{\prime}$ and $g^{\prime} / H^{\prime}$ must be constant functions. It follows that $f+$ $\bar{g}=\alpha H+\beta \bar{H}+\gamma$ for some constants $\alpha, \beta, \gamma$, which is what we needed to prove.

Corollary 7. Let $w$ be positive and $C^{2}$ on an open set $\mathcal{V}$. Then a nontrivial solution to (17) exists if and only if $w=\left|H^{\prime}\right|^{2}$ and

$$
m=C|H|^{2}+\alpha H+\beta \bar{H}+\gamma
$$

for some complex numbers $\alpha, \beta, \gamma$ and some (possibly multi-valued) analytic function $H$ on $\mathcal{V}$ for which the expression (20) is single-valued and the derivative $H^{\prime}$ does not vanish.

\section{References}

[A] E. Almansi, Sulle integrazione dell'equazione differenziale $\Delta^{2 n}=0$, Ann. Math. Pura Appl., 2 (1898), 1-51. 
[Duff] R.J. Duffin, On a question of Hadamard concerning super-biharmonic functions, J. Math. and Phys., 27 (1949), 253-258.

[DKSS1] P.L. Duren, D. Khavinson, H.S. Shapiro and C.Sundberg, Contractive zero-divisors in Bergman spaces, Pacific J. Math., 157 (1993), 37-56.

[DKSS2] _ Invariant subspaces in Bergman spaces and the biharmonic equation, Michigan Math. J., 41 (1994), 247-259.

[E-Pee] M. Engliš and J. Peetre, A Green's function for the annulus, Annali di Math., Pura Appl. (IV), 171 (1996), 313-377.

[Gar] P.R. Garabedian, A partial differential equation arising in conformal mapping, Pacific J. Math., 1 (1951), 485-524.

[H-jram] H. Hedenmalm, A factorization theorem for square area-integrable analytic functions, J. Reine Angew. Math., 422 (1991), 45-68.

$[\mathrm{H}-\mathrm{OpP}] \_$, Open problems in the function theory of the Bergman space, in "Proceedings of the conference in honour of L. Carleson and Y. Domar", Uppsala, Sweden, 1993.

[H-Duke] A computation of Green functions for the weighted biharmonic operators $\Delta|z|^{-2 \alpha} \Delta$, with $\alpha>-1$, Duke Math. J., 75 (1994), 51-78.

[H-BVP] , Boundary value problems for weighted biharmonic operators, Algebra i Analiz, 8 (1996), 173-192.

[Loe] C. Loewner, On generation of solutions of the biharmonic equation in the plane by conformal mappings, Pacific J. Math., 3 (1953), 417-436.

[NS] M. Nakai and L. Sario, Duffin's function and Hadamard's conjecture, Pacific J. Math., 75 (1978), 227-242.

[ShT] H.S. Shapiro and M. Tegmark, An elementary proof that the biharmonic Green function of an eccentric ellipse changes sign, Siam Review, 36 (1994), 99-101.

[Shi1] S.M. Shimorin, Factorization of analytic functions in weighted Bergman spaces, Algebra i Analiz, 5 (1993), 155-177 (in Russian).

[Shi2] - On a family of conformally invariant operators, Algebra i Analiz, 7 (1995), 133-158 (in Russian).

[Ste] M. Stessin, An extension of a theorem of Hadamard and domination in the Bergman space, J. Funct. Anal., 115 (1993), 212-226.

Received October 4, 1995.

MU AV CR

ZITNA 25

11567 PRAGUe 1

CZECH Republic

E-mail address: englis@math.cas.cz

Note: The References to Shimorin's PAPERs on PAge 344 Were GIVEN IN The PRINTED VERSION AS [SH1] AND [SH2]. 